\title{
METHOD FOR MEASURING DISTORTION IN WIDE-ANGLE VIDEO CHANNELS
}

\author{
Aleksander Krotov* Dmitry Volkov Nikolay Romanov Nikolay Gryaznov Evgeny Sosnov \\ Dmitry Goryachkin \\ St. Petersburg Electrotechnical University (“LETI”), The Russian State Scientific Center for Robotics and
Technical Cybernetics (RTC), Russian Federation
}

Methods for correcting aberrations of images, obtained by an optoelectronic camera with the use of preliminary measured calibration function, provoke wide interest in recent years. For the most part these methods are not characterized by high accuracy and are typically used for cameras with objectives having rather high relative distortion. In this article the method is discussed for measuring distortion of a wide-angle objective for calibration of photoelectric cameras designed to measure angular coordinates of remote objects with the accuracy as high as 10 arcseconds. It is proposed to use an air mirror-wedge as a test object, which allows creating a sheaf of collimated beams. The calibration function of a photoelectric camera is calculated by mathematical post-processing of the recorded frames. The proposed method was experimentally tested for photoelectric cameras with full angular fields-of-view of 21 degrees and angular pixel sizes of 20 arcseconds. The subpixel accuracy of determining the calibration function is demonstrated.

Key words: Distortion, Radial distortion, Numerical optimization, Image correction, High accuracy, Calibration function

\section{INTRODUCTION}

For high precision measurements of remote objects angular coordinates, in particular for astronomical measurements with using photoelectric registration, it is necessary to take into consideration the image aberrations resulted from imperfect optics at the stage of recorded frames processing. The significant contribution to image aberration, especially for wide-angle lenses, results from distortion of optical systems.

A method for in-laboratory distortion measurement of wide-angle cameras for electronic theodolites is considered in this paper. To create a test light source it is proposed to use an air mirror-wedge (AMW) formed by two plane-parallel plates. The method is characterized by universality, simplicity and efficiency, as well as by the low cost of the equipment necessary for its implementation.

\section{LITERATURE REVIEW}

To take into account the distortion, a camera consisting of a wide-angle objective and a photoelectric array is typically calibrated using a test light source whose angular coordinates of the elements are known with the required accuracy. There are a lot of investigations are devoted to measuring the image distortion [01-15]. Most of them deal with calibration of photoelectric cameras for surveying at a finite distance, and are not intended for accurate measurements of angular coordinates.

The most popular methods of distortion measurement are usually designed to investigate sufficiently large geometric distortions of the image (more than $1 \%$ ) with a rel- atively small accuracy $[01,02,04-10,13-15]$ or to measure distortion with a high accuracy, but only for narrow field-of-view optical systems [03].

In this article the method is considered for calibration of wide field-of-view video channels for electronic theodolites, intended for measurements of angular coordinates with the accuracy better than 10 arcseconds. The A test light source in this case should consist of a set of point sources located in the far-field zone. Their angular coordinates should be a priori known with the accuracy not worse than 2-3 arcseconds. The point sources should fill totally the field-of-view of the video channel.

The most commonly used test object for calibration of wide-angle cameras intended for measuring the angular coordinates of remote objects is the stellar sky [16]. The calibration based on the stellar sky survey must be carried out in the field at night and requires using of high precision clocks and accurate positioning of the calibrated camera.

The method considered in this article provides a high-precision and simple measurement of distortion even in orthoscopic optical systems with a basic level of relative distortion not exceeded fractions of a percent. A description of the method and experimental results of distortion measurement of wide-angle cameras with an angular field-of-view up to 21 degrees (full angle) and relative distortion at edges of the field-of-view not exceeded 0.3 percent are presented below. 


\section{MATERIALS AND METHODS}

The optical schematic for distortion measurement of wide-angle photoelectric cameras is shown in Figure 1. AMW 4, formed by two plane-parallel plates is used as a test light source. The angle between the plates is $\alpha$. The interior plate surfaces are covered with partially reflective coatings with a reflection coefficient of 85 percent in the operating spectral range. The outer surfaces of the plates are antireflection coated.

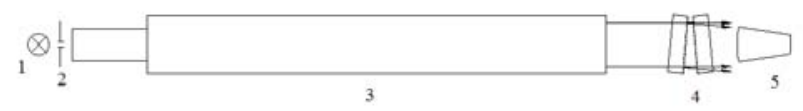

Figure 1: Optical schematic for distortion measurement of wide-angle photoelectric cameras.

$$
\begin{gathered}
1 \text { - light source, } \\
2 \text { - diaphragm, } \\
3 \text { - collimator, } \\
4 \text { - air mirror-wedge, } \\
\text { 5-tested photoelectric camera }
\end{gathered}
$$

The beam with a plane wave front formed by collimator 3 falls onto the AMW. A sheaf of equidistant collimated beams separated by the angles equal to double wedge angle $\alpha$ is formed at the AMW output.

In the focal plane of the photoelectric camera objective (video channel), the beam sheaf is imaged as a chain of focal spots ("star track"). The focal spot corresponding to the non-deflected beam is located near the point of intersection of the optical axis of the objective and the focal plane. When the video channel or the AMW is rotated around the axis, the orientation of the "star track" in the frame changes. Thus, several frames are recorded to fill uniformly the field-of-view of the photoelectric camera with star tracks, as shown in Figure 2.

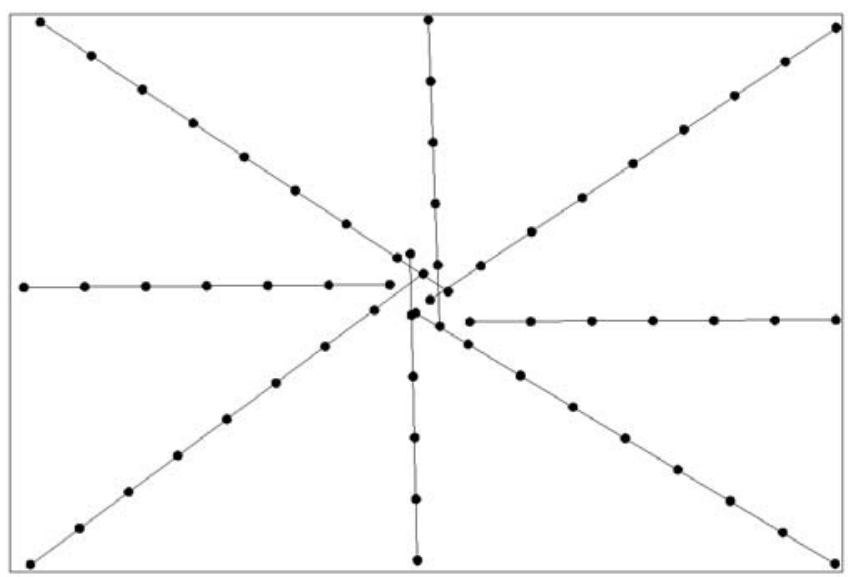

Figure 2: Filling field of view of the photoelectric camera (video channel) with "star tracks".

A straight line for clarity connects the focal spots of each of the tracks
For calibration of the video channel during the subsequent processing of the recorded frames the coordinates of pixels corresponding to the energetic gravity centers of the "star track" focal spots for each frame are calculated. Also, angle of the "star track" slope $y$ relative to horizontal axis $\mathrm{X}$ of the matrix is calculated in the frame by the least squares method.

To calculate the radial distortion, a seventh-order distortion model was used. In "pixel" units of measurement, this model can be written in the following form:

$$
N_{d}=N_{u} \cdot\left(1+K_{3} \cdot N_{u}^{2}+K_{5} \cdot N_{u}^{4}+K_{7} \cdot N_{u}^{6}\right)^{1)}
$$

where $N_{d}$ is the "measured" (distorted) distance from the gravity center of the focal spot to the camera axis, $N_{u}$ is the "undistorted" position of the focal spot $K_{3}, K_{5}, K_{7}$ are respectively the third, fifth and seventh order distortion coefficients.

The "measured" distance $N_{d}$ is related to the video channel parameters by the following ratio:

$$
N_{d}=\sqrt{\left(X_{m}-X_{c}\right)^{2}+\left(Y_{m}-Y_{c}\right)^{2}}
$$

where $X_{m}, Y_{m}$, are the measured values of the coordinates of the gravity center pixel of $\mathrm{m}^{\text {th }}$ focal spot $X_{c}, Y_{c}$, are the coordinates of the camera axis pixel.

The following ratio relates the "undistorted" position of the focal spot $N_{u}$ to the video channel parameters:

$$
N_{u}=\frac{F \cdot \tan (\theta)}{d_{p i x}}
$$

Here $F$ is the focal distance of the video channel, $\theta$ is the angle between the axis of the objective and the axis of the given sheaf beam, $d_{\text {pix }}$ is the pixel size.

Since aberrations introduced by distortion are negligibly small for the non-deflected beam in the sheaf (the first beam), angle $\theta_{0}$ between the axis of the objective and the axis of this beam is related to the coordinates of the gravity center of the spot and the parameters of the video channel, as follows:

$$
\begin{aligned}
& \tan \left(\theta_{x 0}\right)=\frac{\left(x_{d 0}-x_{c}\right) \cdot d_{p i x}}{F} \\
& \tan \left(\theta_{y 0}\right)=\frac{\left(Y_{d 0}-Y_{c}\right) \cdot d_{p i x}}{F} \\
& \tan \left(\theta_{0}\right)=\sqrt{\tan ^{2}\left(\theta_{x 0}\right)+\tan ^{2}\left(\theta_{y 0}\right)^{6)}}
\end{aligned}
$$

where $X_{d 0}, Y_{d 0}$, are the measured coordinates in pixels of the gravity center of the focal spot for the first beam. 
The coordinate system of the video channel is defined as follows: $X$-axis of the video channel is parallel to $X$-axis of the matrix, $Y$-axis of the video channel is parallel to $Y$-axis of the matrix, Z-axis of the video channel coincides with the axis of symmetry of the objective (the radial distortion axis).

The unit vector of the axis direction of $j^{\text {th }}$ beam in the sheaf in the coordinate system of the video channel is determined by the formula:

$$
\begin{aligned}
& K_{j}= \\
& \left.\left(\begin{array}{c}
\cos (\gamma) \cdot \sin (2 \cdot(j-1) \cdot \alpha-\psi)-\sin (\gamma) \cdot \sin (\varphi) \cdot \cos (2 \cdot(j-1) \cdot \alpha-\psi) \\
\sin (\gamma) \cdot \sin (2 \cdot(j-1) \cdot \alpha-\psi)-\cos (\gamma) \cdot \sin (\varphi) \cdot \cos (2 \cdot(j-1) \cdot \alpha-\psi) \\
\cos (\varphi) \cdot \cos (2 \cdot(j-1) \cdot \alpha-\psi)
\end{array}\right) 7\right)
\end{aligned}
$$

Here $y$ is the angle of inclination of the "star track" in the frame relative to $X$-axis of the matrix, $\psi$ is the angle between the first beam and the projection of the axis of the photoelectric camera on the plane in which the sheaf of beams lies (sheaf plane), $\varphi$ is the angle between the axis of the video channel (the axis of the objective) and the sheaf plane.

The angles of orientation of the sheaf plane relative to the camera's coordinate system $(\psi$ and $\varphi)$ are related to the coordinates of the first beam and the orientation of the "star track" in the frame with the following ratios:

$$
\begin{aligned}
& \tan (\varphi)=\cos (\gamma) \cdot \tan \left(\theta_{y 0}\right)-\sin (\gamma) \cdot \\
& \tan \left(\theta_{x 0}\right) \\
& \tan (\psi)= \\
& -\frac{\cos (\gamma) \cdot \tan \left(\theta_{x 0}\right)+\sin (\gamma) \cdot \tan \left(\theta_{y_{0}}\right)}{\sqrt{1+\left(\cos (\gamma) \cdot \tan \left(\theta_{y_{0}}\right)-\sin (\gamma) \cdot \tan \left(\theta_{x_{0}}\right)\right)^{2}}}
\end{aligned}
$$

Finally, the angle between the axis of the $\mathrm{j}^{\text {th }}$ beam in the sheaf and the axis of the video channel is given by:

$$
\cos \left(\theta_{j}\right)=K_{j_{z}}
$$

To find the camera parameters and distortion coefficients by the Levenberg-Marquardt method, the optimization problem for equation (1) is solved for each focal spot from each frame of "star track" recording with taking into account the interrelation of the quantities described by expressions $(2-10)$. The most probable values of the following parameters are determined: the focal length of the camera $F$, pixel coordinates of the axis of the video channel $X_{c}, Y_{c}$, (the symmetry axis of the distortion function), and the third, fifth and seventh order distortion coefficients $K_{3}, K_{5}, K_{7}$.

\section{RESULTS}

Experimental measurements of the distortion according to the presented method were carried out with video channels of two types differing by image sensors and objectives $[17,18]$. Both objectives were specially designed for use in electronic theodolites, and therefore they were subjected to high demands in terms of distortion correction and uniformity of an image quality throughout the field, including corners of the frame. Technical characteristics of video channels, including parameters of video sensors and optical objectives, used in the investigated video channels, are given in Table 1.

For both objectives the distortion was corrected almost completely and even in the corners of the frame it did not exceed 0.3 percent. The difference between the paraxial and real position of the image of any point did not exceed $20 \mu \mathrm{m}$ (almost along the full field its value ranges from 0 to $8 \mu \mathrm{m})$. This is less or comparable to the size of a single pixel of the image sensor, which was $7.4 \mu \mathrm{m}$ in video channel 1 , and $5.5 \mu \mathrm{m}$ in video channel 2. Below, in Figure 5, the calculated distortion function is given with the ideal accuracy of assembling the objectives.

At achieved level of distortion correction a high-precision measurement of the geometrical distortions of the optical system should be made taking into consideration the contribution of asymmetric aberrations of broad beams, as well as inaccuracy in the components manufacture, and errors in assembly and alignment of the both objectives and video channels.

Considering this, in the both objectives special attention was paid to minimizing coma aberrations and field curvature, and their design included adjustment movements of the most sensitive optical components, which made it possible to achieve uniformity and high resolution in the video channels over the full frame. The curves of polychromatic modulation transfer function (MTF) of the objectives are shown in Figure 3.

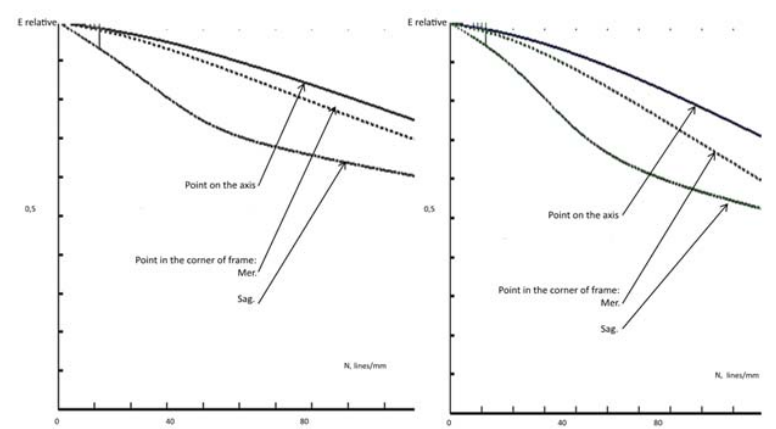

Figure 3: MTF of video channel No.1 lenses (left) and video channel No.2 (right) 
The test sheaf of beams was created with the use of a collimator from a standard optical bench (focal length $1600 \mathrm{~mm}$, light diameter $150 \mathrm{~mm}$ ) and an AMW. The AMW was formed by two plane-parallel plates of BK7 glass with the diameter of $80 \mathrm{~mm}$ and the surface optical quality $\mathrm{N}=0.1, \Delta N=0.1$. Plates were assembled in a mount, which provided possibility to adjust the angle between the plates and to fix them rigidly after that.

The maximum number of beams in the sheaf was limited by the optical quality of the surfaces of the AMW plates. Multiple reflections from mirror surfaces of the AMW result in distortions of the beam wavefront, which arise due to surfaces imperfectness and accumulate (practically add up together). For the AMW unit used in our experiments, the angular divergence of the beam after 9 reflections (beam number 9) turns out to be unacceptably large. Therefore, the value of AMW angle was chosen so that a half angle of the field of view of the video channel comprises nine beams (that is, there are nine focal spots in the half image diagonal). For example, to measure the distortion of video channels No. 2, the angle between the plates was chosen about 40 arcminutes. The AMW angle was measured using a goniometer with the accuracy not less than 0.5 arcsecond.

Note that the AMW must be set in such a way that its edge should be perpendicular to the input beam axis. Deviation from the perpendicularity $\delta \varphi$ will lead to error $\delta \alpha$ in angle $2 \cdot \alpha$ between the adjacent beams so that $\delta \varphi \sim \sqrt{\frac{\delta \alpha}{\alpha}}$. As estimations show, the requirements on the perpendicularity are not rigid. For example, if $\delta \alpha=0.1$ arcsecond and $\alpha=40$ arcminutes, the permissible deviation in the AMW edge perpendicularity to the input beam axis is $\delta \varphi \sim 22$ arcminutes. During the measurement of the distortion of video channels the edge of the AMW was set perpendicular to the axis of the incident beam using the autocollimator with the accuracy better than 10 arcseconds.

For each video channel the eight successive frames were recorded with different orientations of the "star track" in the frame field in accordance with Figure 2. As indicated above, the following values were calculated as a result of mathematical processing of these frames: camera focal length $F$, pixel coordinates $X_{c}, Y_{c}$ of the video channel axis (axis of symmetry of the distortion function) and third, fifth and seventh order distortion coefficients $K_{3}, K_{5}, K_{7}$ (see 1-10).

Figure 4 shows an example of the results of the distortion measurement for one of the video channels 2 .

The solid curve represents the calibration function of the video channel (distortion function ) calculated using ratio:

$$
\begin{aligned}
& D_{c a l}(\theta)=K_{3} \cdot\left(\frac{F \cdot \tan \theta}{d_{y i x}}\right)^{3}+K_{5} \cdot\left(\frac{F \cdot \tan \theta}{d_{y i x}}\right)^{5}+K_{7} . \\
& \left(\frac{F \cdot \tan \theta}{d_{y i x}}\right)^{7}
\end{aligned}
$$

The points in Figure 4 correspond to focal spots of the sheaf of beams and show the measured value of the distortion $D_{\text {exp }}(\theta)$ for each focal spot. The position of each point was calculated using ratio:

$$
\left.D_{\text {exp }}(\theta)=\sqrt{\left(X_{m}-X_{c}\right)^{2}+\left(Y_{m}-Y_{c}\right)^{2}}-\frac{F \cdot \tan \theta}{d_{p i x}} 13\right)
$$

The root-mean-square deviation of the measured distortion values of the focal spots from the calibration function of the video channel shown in Figure 4 was 0.098 pixels.

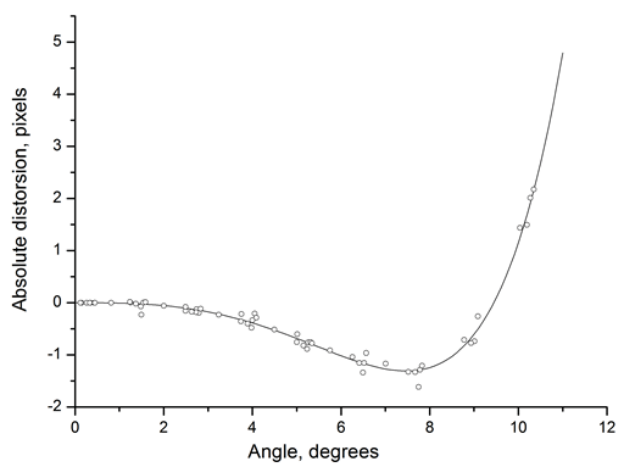

Figure 4: The calibration function of the video channel (distortion function, solid line) calculated according to the results of measurements. The circles show positions of the focal spots of the "star tracks"

\section{DISCUSSION}

As mentioned above, the proposed method was verified by calibration of several wide-angle video channels developed by the authors of this paper. Figure 5 shows comparison of the measurement results and the theoretical function of distortion.

In Figure 5 the solid curve shows the distortion function for the objective with the ideal accuracy of assembling. The dotted lines show the calibration functions obtained from the results of measurements for 15 video channels No. 2.

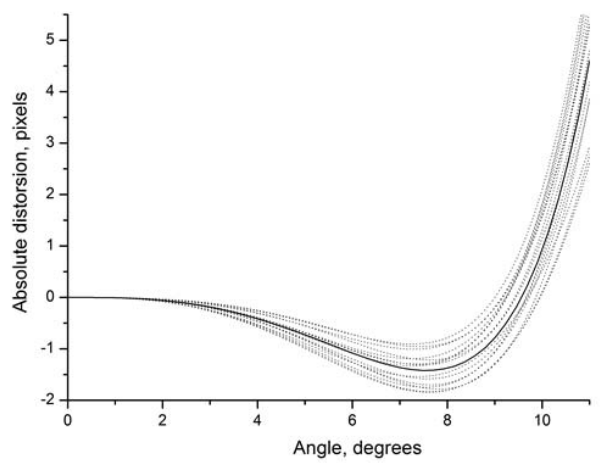

Figure 5: Distortion function (solid line) calculated for ideal assembling the objective. Dotted lines show calibration functions for 15 video channels 
Table 1: Technical specifications of video channels

\begin{tabular}{|c|c|c|}
\hline Technical specifications & Video channel No. 1 & Video channel No.2 \\
\hline Video Sensor & KAI-4021 IMAGE SENSOR & KAI-08051 IMAGE SENSOR \\
\hline Number of Active Pixels & $2048 \times 2048$ & $3296 \times 2472$ \\
\hline Pixel Size & $7.4 \mu \mathrm{m} \times 7.4 \mu \mathrm{m}$ & $5.5 \mu \mathrm{m} \times 5.5 \mu \mathrm{m}$ \\
\hline Linear size of the image receiver, $\mathrm{mm}$ & $15.15 \times 15.15$ (diagonal 21.43) & $18.13 \times 13.60$ (diagonal 22.66) \\
\hline Focal length of the objective, $\mathrm{mm}$ & 80 (objective17) & 60.1 (objective18) \\
\hline Angular pixel size, arcsec & $19^{\prime}$ & $18.75^{\prime}$ \\
\hline The largest diameter of the entrance pupil, mm & 61 & 46 \\
\hline f-number & $\mathrm{f} / 1.3$ & $680-820$ \\
\hline Spectral range, $\mathrm{nm}$ & $680-820$ & 21.3 \\
\hline Field-of-view, degree & 16 & $8 / 7$ \\
\hline Number of elements/groups & $8 / 7$ & $134 / 56$ \\
\hline Length / diameter, $\mathrm{mm}$ & $150 / 68$ & \\
\hline
\end{tabular}

NOMENCLATURE

\begin{tabular}{|c|c|c|}
\hline \multicolumn{3}{|c|}{ Abbreviation } \\
\hline AMW & \multicolumn{2}{|l|}{ Air mirror-wedge } \\
\hline MTF & Modulation transfer function & \\
\hline \multicolumn{2}{|r|}{ Description } & Unit of measure \\
\hline$\alpha$ & angle of the air mirror-wedge & rad \\
\hline $\mathrm{Y}$ & angle of the "star track" slope & rad \\
\hline$N_{d}$ & "measured" (distorted) distance in "pixel" units & \\
\hline$N_{u}$ & "undistorted" position of the focal spot in "pixel" units & \\
\hline$K_{3}, K_{5}, K_{7}$ & third, fifth and seventh order distortion coefficients & \\
\hline$X_{m}, Y_{m}$ & measured coordinates of the spot gravity center in pixels & \\
\hline$X_{c}, Y_{c}$ & coordinates of the axis in pixels & \\
\hline$F$ & focal distance of the video channel & $\mathrm{mm}$ \\
\hline$\theta$ & angle between the axis of the objective and the axis of the beam & rad \\
\hline$d_{p i x}$ & pixel size & $\mathrm{mm}$ \\
\hline$\theta_{0}$ & $\begin{array}{c}\text { angle between the axis of the objective and the axis of the first beam of the } \\
\text { sheaf }\end{array}$ & rad \\
\hline$X_{d 0}, Y_{d 0}$ & measured coordinates in pixels of the focal spot for the first beam & \\
\hline$\Psi$ & $\begin{array}{l}\text { angle between the first beam and the projection of the axis of the objective on } \\
\text { the sheaf plane }\end{array}$ & rad \\
\hline$\varphi$ & angle between the axis of the objective and the sheaf plane & $\mathrm{rad}$ \\
\hline$\theta_{j}$ & angle between the axis of the $j^{\text {th }}$ beam in the sheaf and the axis of the objective & $\mathrm{rad}$ \\
\hline $\mathrm{Kj}$ & unit vector of the axis direction of $\mathrm{j}^{\text {th }}$ beam & \\
\hline$\delta \varphi$ & deviation from the perpendicularity of the input beam axis to AMW edge & \\
\hline$\delta \alpha$ & error in angle $2 \cdot \alpha$ between the adjacent beams of the sheaf & \\
\hline$D_{\text {cai }}(\theta)$ & calibration function of the video channel & \\
\hline$D_{\operatorname{sxp}}(\theta)$ & measured value of the distortion & \\
\hline
\end{tabular}


As can be seen, the maximum deviations of the measured calibration functions of the video channels from the calculated distortion function are about 0.5 pixels, that corresponds to $\approx 10$ arcseconds in terms of angular units and less than $3 \mu \mathrm{m}$ in linear units. Apparently, these differences are associated with errors in the manufacture of optical components and an inaccuracy in assembling the system within the tolerances of the mutual position of the optical components of the system. As the Monte-Carlo calculations showed, within allowable deviations in the assembly accuracy the measured distortion positions of the focal spots in the photodetector plane differ from the calculated ones by values not exceeding $3 \mu \mathrm{m}$.

As follows from the results of measurements of the distortion function, the proposed method makes possible to determine the calibration function with the sub-pixel accuracy. The AMW allows to use both monochromatic and broadband light sources for channel calibration.

\section{CONCLUSION}

The method, proposed in the paper, makes it possible to derive theoretically and experimentally the calibration function for wide-angle video channels with objectives having small relative distortion. The calibration function derived in such a way can be used for image correction as well as for precise calculation of angular coordinates of the targets in a frame.

The method is characterized by universality, simplicity and efficiency, as well as by the low cost of the equipment necessary for its implementation.

It is worth noting that the method proposed fits objectives with the symmetric function of distortion. There are precisely those wide-angle objectives of sufficiently high optical quality, which are used in electronic theodolites.

\section{ACKNOWLEDGMENTS}

This article has been prepared with the help of the financial support of the Ministry of Education and Science of the Russian Federation. Agreement No.14.581.21.0018 dated 9 November 2015; Unique Identifier for the Applied Scientific Research and Development is RFMEFI58115X0018.

\section{REFERENCES}

1. GOST 20825-75. Objectives for photography. Method of measuring distortion. (1976), from http://docs. cntd.ru/document/1200015646, accessed on 201801-23.

2. Zhimbueva, L.D. (n.a.). Method of determination of summary distortion of digital images. Computer Optics, vol. 1, no. 3, 347-355.

3. Kozhin, A.V., Zavyazkin, V.F., Silant'eva, N.S. (2006). Method of determination of distortion of long-focus objectives. PatentRU No.2276778. JSC "Krasnogorsky Zavod", Krasnogorsk, from http:// www.freepatent.ru/patents/2276778, accessed on 2017-11-21.

4. Cattaneo, C., Mainetti, G., Sala, R. (2015). The importance of camera calibration and distortion correction to obtain measurements with video surveillance systems. Journal of Physics: Conference Series, vol. 658, no. 1, DOI: 10.1088/17426596/658/1/012009, from https://www.researchgate. net/publication/284204844_The_Importance_ of_Camera_Calibration_and_Distortion_Correction_to_Obtain_Measurements_with_Video_Surveillance_Systems? sg=JohWRHIkQ8VHPkRhmh2JGXIcn4SadAHMBnCQkYizrrq-42ZAIIYi7S4Yk e2oXDHaCifHMvfN7VzriY, accessed on 2017-12-20.

5. Reznicek, J. (2014). Method for measuring lens distortion by using pinhole lens. The International Archives of the Photogrammetry, Remote Sensing and Spatial Information Sciences, vol. XL-5, no. 5, 509-515, DOI: 10.5194/ isprsarchives-XL-5-509-2014, from https://www. researchgate.net/publication/274676151_Method_for_Measuring_Lens_Distortion_by_Using_Pinhole_Lens?ev=publicSearchHeader\&_sg=BiBSOsMJKgrHbyy1XmfN6sTGI7wbu8QADfKwGcZ6lke9ZkgJALD-yGXc9gQxLbAeRaMQeKmUy3BI7dQ, accessed on 2017-12-25.

6. Cheng, T.-H. (2004). Method for calibration and correction of radial lens distortion, from https://patents. google.com/patent/WO2003043308A2, accessed on 2017-11-23.

7. Remondino, F., (2006). Digital camera calibration methods: considerations and comparisons. ISPRS Commission V Symposium "Image Engineering and Vision Metrology”, vol. XXXVI, part 5, p. 266-272, from http://close-range.com/docs/Digital_camera calibration_methods_10.1.1.67.8805.pdf, accessed on 2017-11-24.

8. Lin, T.-W., Chang, C.-Y. (2009). Enhanced calibration method for camera distortion. ICCAS-SICE 2009, p. 1115-1120. 
9. Souchard, C. (2011). Estimating and removing lens distortion from scenes. US $20070098296 \mathrm{~A} 1$, from http://patft.uspto.gov/netacgi/nph-Parser?Sect1=PTO1\&Sect $2=$ HITOFF \&d=PALL \&p=1\&u=\%2Fne tahtm I \% 2 FP T O \% $2 \mathrm{Fs} \mathrm{rchnum}$. h t $\mathrm{m} \& \mathrm{r}=1 \& \mathrm{f}=\mathrm{G} \& \mathrm{l}=50 \& \mathrm{~s} 1=7,912,317 . \mathrm{PN} . \& O S=P N / 7,91$ 2,317\&RS=PN/7,912,317, accessed on 2018-04-11.

10. Tang, Zh., Grompone von Gioi, R., Monasse, P., Morel, J.-M. (2012). High-precision camera distortion measurements with a "calibration harp". Journal of the Optical Society of America, vol. 29, no. 10, 21342143, DOI: 10.1364/JOSAA.29.002134, from https:// www.researchgate.net/publication/233827976_ High-precision_camera_distortion_measurements with_a_calibration_harp?_sg=ONonP9P_B-XjpJbeDazL08dA7D-HsOT-uRnRFnFebvhqZXwYYpEQBZKAE1FxH1u7LZ6WXmtkAvmCRxfQ, accessed on 2018-02-19.

11. Huang, C.-W., Chou, C.-M., Lin, T.-W., Chang, C.-Y. (2013). Adaptive calibration method for camera distortion. Journal of Vibration and Control, vol. 19, no. 1, 86-93, DOI: 10.1177/1077546311433915.

12. Villiers de, J.P., Leuschner, F.W., Geldenhuys, R. (2008). Centi-pixel accurate real-time inverse distortion correction. 2008 International Symposium on Optomechatronic Technologies. SPIE, DOI: 10.1117/12.804771, from http://researchspace.csir. co.za/dspace/bitstream/handle/10204/3168/De\%20 Villiers_2008.pdf;jsessionid=8225D91FFB4F83483F8EACBFAEC67031? sequence $=1$, accessed on 2018-03-09.

13. Wang, A., Qiu, T., Shao, L. (2009). A simple method of radial distortion correction with centre of distortion estimation. Journal of Mathematical Imaging and Vision, vol. 35, no. 3, 165-172, DOI: 10.1007/s10851-009-0162-1, from https://www. researchgate.net/publication/220146291_A_Simple_Method_of_Radial_Distortion_Correction_with_ Centre_of_Distortion_Estimation?ev=publicSearchHeader\&_sg=ZC-yxkCHBQNdXXgFymjkD9hBVIuxYufw2EXynpu67xcaQF0ejjXNtANda4ii8bsctNicRg6nazNVasg, accessed on 2018-03-01.
14. Zhang, Z., Matsushita, Y., Ma, Y. (2014). Camera calibration with lens distortion from low-rank textures. Patent: US8818132B2, from http://perception. csl.illinois.edu/matrix-rank/Files/calibration.pdf, accessed on 2018-03-27.

15. Wenzel, K., Ábrahám, G., Tamás, P., Urbin, Á. (2015). Measurement of distortion using the moiré interferometry. Optics, vol. 4, no. 3-1, 14-17, DOI: 10.11648/j.optics.s.2015040301.14, from http://article.sciencepublishinggroup.com $/ \mathrm{html} / 10.11648$.j.optics.s.2015040301.14.html, accessed on 2018-0319.

16. Kurkov, V.M. (1980). Methods of recording systematic aberrations of an aero photograph. Selfcalibration. Izvestiya Vuzov. Geodezy and Aero Photograpy, no. 6, 75-79.

17. Volkov, D.Yu., Gryaznov, N.A., Kovalev, I.A., Sosnov, E.N. (2017). High luminosity objective. Patent on useful model RU No.169945 as of 07.04.2017.

18. Volkov, D.Yu., Gryaznov, N.A., Kovalev, I.A., Sosnov, E.N. (2017). High luminosity objective. Patent on useful model RU No.169342 as of 15.03.2017.

\section{AUTHOR'S INFORMATION}

${ }^{1}$ The Russian State Scientific Center for Robotics and Technical Cybernetics (RTC).

${ }^{2}$ The Russian State Scientific Center for Robotics and Technical Cybernetics (RTC).

${ }^{3}$ The Russian State Scientific Center for Robotics and Technical Cybernetics (RTC).

${ }^{4}$ The Russian State Scientific Center for Robotics and Technical Cybernetics (RTC).

${ }^{5}$ The Russian State Scientific Center for Robotics and Technical Cybernetics (RTC).

${ }^{6}$ The Russian State Scientific Center for Robotics and Technical Cybernetics (RTC).

${ }^{7}$ Saint Petersburg Electrotechnical University "LETI". 